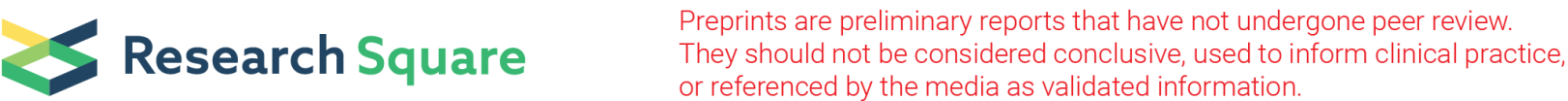

\section{Alterations in the Histological Features of the Intestinal Mucosa in Malnourished Adults of Bangladesh}

Md. Shabab Hossain ( $\nabla$ dr.shabab@icddrb.org)

International Centre for Diarrhoeal Disease Research

S. M. Khodeza Nahar Begum

Bangladesh Specialized Hospital

M Masudur Rahman

Dhaka Medical College and Hospital

Ramendra Nath Mazumder

International Centre for Diarrhoeal Disease Research

Mashud Parvez

Bangladesh Specialized Hospital

Md. Amran Gazi

International Centre for Diarrhoeal Disease Research

Md. Mehedi Hasan

International Centre for Diarrhoeal Disease Research

Shah Mohammad Fahim

International Centre for Diarrhoeal Disease Research

Subhasish Das

International Centre for Diarrhoeal Disease Research

Mustafa Mahfuz

International Centre for Diarrhoeal Disease Research

Shafiqul Alam Sarker

International Centre for Diarrhoeal Disease Research

Tahmeed Ahmed

International Centre for Diarrhoeal Disease Research

\section{Research Article}

Keywords: BEED, EED, Dysfunction, histological

Posted Date: December 7th, 2020

DOI: https://doi.org/10.21203/rs.3.rs-107106/v1 
License: (c) (i) This work is licensed under a Creative Commons Attribution 4.0 International License. Read Full License

Version of Record: A version of this preprint was published at Scientific Reports on January 27th, 2021. See the published version at https://doi.org/10.1038/s41598-021-82079-6. 


\section{Abstract}

There is paucity of knowledge on the histological features of the intestinal mucosa in malnourished adults of Bangladesh. The purpose of the study was to explore the histological features of the intestinal mucosa in malnourished adults of Bangladesh and to compare the findings with their well-nourished counterparts. 64 adults ( 37 malnourished with body mass index, BMK $18.5 \mathrm{~kg} / \mathrm{m}^{2}$ and 27 controls with $B M l>18.5 \mathrm{~kg} / \mathrm{m}^{2}$ ) from the Bangladesh Environmental Enteric Dysfunction (BEED) study, who underwent upper-gastrointestinal endoscopy, were selected for this study. With a view to address the association of environmental enteric dysfunction (EED) with malnutrition, upper-gastrointestinal endoscopy was performed and mucosal biopsies from the distal duodenum were studied for histopathology. Villous height, crypt depth, and presence of inflammatory infiltrates in lamina propria were investigated. Bivariate analysis was performed to quantify the relation between malnutrition and the histological features. About $95 \%$ adults, irrespective of nutritional status, were diagnosed to have chronic non-specific duodenitis on histopathology. Malnourished adults suffered significantly more from chronic active duodenitis compared to their well-nourished counterparts $(p=0.003)$. Malnourished adults also had significantly higher frequency of subtotal villous atrophy, crypt hyperplasia and marked cellular infiltration in the lamina propria than the healthy controls $(p<0.05)$.

\section{Introduction}

The gut is central to health and development and a healthy 'functional' intestine enhances physical and intellectual development. While on the other hand, sub-optimal functioning of the gut leads to poor health ${ }^{1}$. The prevalence of chronic intestinal inflammation is very high in the tropics and it has been recognized that gut structure and function are almost universally abnormal, especially among children living in these regions ${ }^{2}$. Alterations in small bowel function in children of developing countries in their early phases of life result from altered mucosal architecture and inflammation of the intestinal mucosa ${ }^{3}$. These changes are said to be the result of factors related to environmental contamination and persist throughout life. Studies show that the condition has been reversed in non-native foreign individuals returning to their original homelands even after prolonged inhabitation in resource-poor settings suggesting the role of exposure in contaminated environment ${ }^{3-5}$. The altered mucosal architecture and inflammation of the intestinal mucosa is thought to be the consequence of chronic exposure of enteric pathogens through fecal contamination, and is being referred to as environmental enteric dysfunction $(\mathrm{EED})^{3}$.

EED, previously known as environmental enteropathy (EE), predisposes to poor growth in children and malabsorption in people of all ages ${ }^{6}$ and is considered to be one of the major causative factors of malnutrition in this region ${ }^{7}$. The mechanism of EED has been described in many studies ${ }^{8,9}$. As EED is clinically asymptomatic, other than its manifestation as sub-acute weight loss and impaired growth, the traditional gold standard for diagnosis is considered to be intestinal biopsy and studying of the intestinal histology ${ }^{10}$. However, collection of small intestinal biopsy samples is ethically and technically infeasible, 
especially in asymptomatic individuals ${ }^{8}$. As a result, only a few studies conducted in some other countries have investigated this and have found shorter and thickened villi, increased crypt depth and inflammatory cellular infiltration in intestinal biopsy samples to be the hallmarks of EED ${ }^{10}$.

Though EED is acquired during infancy, it also persists in adulthood, and the fact is that it was actually first recognized in the adult population ${ }^{11}$. In general terms, the presentation pattern shifts from stunting to malabsorption induced malnutrition characterized by low body mass index (BMI). Bangladesh, apart from being the eighth-most populous country, is also one of the poorest countries of the world ${ }^{12}$. It is a well-established fact that BMI is an indicator of poverty and has an inverse relationship with socioeconomic status ${ }^{13}$. Studies show that malnutrition rates are very high for male adults in South Asia, almost equal to that of women, and considerably higher than that in Sub-Saharan Africa ${ }^{14}$.

There is insufficient information on intestinal histological characteristics in malnourished adults in Bangladesh. Because of this knowledge gap, we sought to evaluate these parameters in malnourished adults and compare the findings with their well-nourished counterparts.

\section{Methods}

\section{Study site and Data collection}

This study is a part of the Bangladesh Environmental Enteric Dysfunction (BEED) study (ClinicalTrials.gov ID: NCT02812615; Link: https://clinicaltrials.gov/ct2/results? cond=NCT02812615\&term=\&cntry=\&state=\&city=\&dist=; Date of first registration: $24 / 06 / 2016$ ), which is a community-based nutrition intervention study conducted in Dhaka, Bangladesh. The objective of the BEED study was to validate non-invasive biomarkers of environmental enteric dysfunction (EED) with small intestinal biopsy in order to have a better understanding of the disease pathogenesis. In the adult cohort of the study, malnourished adults aged $18-45$ years $(\mathrm{BMl}<18.5 \mathrm{~kg} / \mathrm{m} 2)$ were enrolled. The participants received on-site nutritional intervention for 60 days consisting of one egg, $150 \mathrm{ml}$ of whole milk and micronutrient sprinkles, as well as, nutritional counseling daily, for six days a week. The details and overall design of the BEED study have already been published elsewhere ${ }^{15}$. Participants failing to respond to nutritional therapy and also negative for secondary malnutrition, i.e. tuberculosis, parasitic infection were considered as probable cases of EED and upper-gastrointestinal (UGI) endoscopy was performed. Well-nourished adults (BMl $>18.5 \mathrm{~kg} / \mathrm{m} 2$ ) suffering from functional dyspepsia according to the Rome III criteria who had no evidence of any organic diseases on endoscopy and were otherwise healthy, were enrolled from the Gastroenterology Outpatient Department of Dhaka Medical College and Hospital (DMCH) as controls. For this particular study, a total of 64 adults (37 malnourished, 27 controls) were enrolled from July 2016 to May 2018. The study conforms to the principles outlined in the Declaration of Helsinki. Ethical approval was obtained from the Institutional review committee of icddr,b and all activities were performed in accordance with the relevant guidelines. The protocol number is PR16007. A written informed consent for the intervention as well as UGI endoscopy and biopsy was obtained from the participants after a detailed explanation of the aims and procedures of the study. 


\section{Duodenal biopsy}

UGI endoscopy procedures of malnourished and control groups were performed by endoscopists (RNM and MMR) at icddr,b Dhaka Hospital and Dhaka Medical College Hospital respectively using Olympus CV170 scopes under sedation. Biopsy specimens were taken from the distal part of the duodenum (distal to the ampulla of Vater) using EndoJaw ${ }^{\mathrm{TM}}$ FB-230K $2.8 \mathrm{~mm}$ disposable biopsy forceps (Olympus Medical Systems Corporation, Tokyo, Japan). For fixation, biopsy samples were immediately placed in vials containing $10 \%$ buffered formalin solution and paraffin sections were prepared and stained by hematoxylin and eosin (H\&E) stain. All the biopsies were reviewed by expert pathologists (KNB and MP), blinded to the case histories and endoscopic findings. The villous height, crypt depth, presence and intensity of inflammatory infiltrates in lamina propria and intraepithelial lymphocytes (IELs) were determined from the biopsied specimens ${ }^{16}$.

\section{Duodenitis and intestinal histological characteristics}

An operational classification was developed based on pathogenesis and cellular activity (Fig. 1). Nonspecific duodenitis was defined as inflammation and morphological alteration of the duodenal mucosa not associated with any other pathological process ${ }^{17}$. Whereas, specific or secondary duodenitis referred to presence of a disease process such as Crohn's disease, sarcoidosis, etc ${ }^{18}$. Whether the duodenitis was active (chronic active duodenitis) or not (chronic duodenitis) was based on the presence of neutrophilic infiltration or polymorphonuclear invasion, characterized by epithelial degeneration and regeneration with intercellular edema ${ }^{19}$.

As reported in several studies $2,6,9,20,21$, morphometric parameters of intestinal histology included measures of villous and crypt remodeling and infiltration of inflammatory infiltrates in the lamina propria. Villous changes included villous blunting or atrophy, which was defined as flattening of the mucosal surface secondary to the shortening and blunting of the intestinal villi and changes in crypt referred to elongation or hyperplasia of the crypts and was defined as an increase in the length of crypts and a reduction in the normal crypt to villous $(\mathrm{C}: \mathrm{V})$ ratio $^{22}$. Total villous atrophy was defined as complete atrophy or flattening of the villi with a $\mathrm{V}: \mathrm{C}$ ratio varying from $0: 1$ to $1: 1$, whereas subtotal and mild villous atrophy referred to partial blunting or shortening of the villi and mild reduction of villous height than normal respectively ${ }^{22}$. An increased IEL count in an otherwise normal duodenal biopsy specimen may be associated with certain immunological disorders, use of NSAIDs, lymphocytic/ collagenous colitis, bacterial overgrowth and gluten sensitive enteropathy ${ }^{22}$.

Even in the absence of active inflammation, the lamina propria might consist of plasma cells and lymphocytes ${ }^{22}$, and slides containing the presence of an excess of these infiltrates than normal based on eye estimation were only taken into consideration. As judged by the eye, presence of inflammatory infiltrates was further categorized into marked, moderate and mild forms. A marked infiltration denoted an intense and diffuse inflammatory infiltration clearly distinguishable on naked eye, a moderate infiltration denoted the presence of a lymphoid aggregation or follicle and a mild infiltration denoted a 
higher number of infiltrates than normal. More than 30 IELs/100 enterocytes was diagnosed as intraepithelial lymphocytosis ${ }^{22}$. IEL count was done by counting the number of lymphocytes per 20 enterocytes present on a random villous tip, and then summing up the numbers of these lymphocytes from such 5 random villi ${ }^{22}$. That is, 20 enterocytes multiplied by 5 villi resulting in the number of lymphocytes per 100 enterocytes. Subjective morphologic analysis of the mucosal surface architecture was carried out in LEICA DM 1000 LED microscope. Fig. 2 shows the schemes used to assess the morphological alterations of intestinal mucosa.

\section{Statistical analyses}

Statistical analyses were performed using SPSS version 20.0 (IBM). Mean values, standard deviation (SD) and 95\% confidence intervals ( $\mathrm{Cl})$ of means were used to describe the distribution and prevalence. Bivariate analysis was performed to quantify the relation between malnutrition and histological features.

\section{Results}

A total of 64 adults (37 malnourished, 27 controls) with mean age of $26 \pm 7$ years underwent UGI endoscopy. Among them, 39 (40.9\%) adults were female.

Majority of the adults from both malnourished and control groups had chronic non-specific duodenitis on histopathology. The histological features of malnourished adults significantly pointed towards an active inflammation, which is chronic active duodenitis $(p=0.003)$. However, the inclination was significantly more towards the non-active form of chronic duodenitis $(p=0.007)$ in controls. There were 2 cases from the malnourished cohort who had specific duodenitis, one of them having diagnosed as celiac disease and the other with immune-proliferative small intestinal disease (IPSID). A small number of malnourished adults also had raised IEL count. Table-1 shows the distribution of duodenitis based on the operational classification.

Majority of the adults from the control group had normal villous height (20/27) as well as crypt depth (22/27). On the other hand, $24 \%$ (9/37) of the malnourished cases had subtotal villous atrophy, $14 \%$ $(5 / 37)$ of the malnourished cases had total villous atrophy and $68 \%(25 / 37)$ of the malnourished cases had crypt hyperplasia. Representative histological images of normal villous architecture, mild villous atrophy, subtotal villous atrophy and total villous atrophy with crypt hyperplasia obtained using hematoxylin and eosin (H\&E) stain are shown in Fig. 3a, b, c and d respectively. Though mild lymphocytic infiltration was present in more than half $(14 / 27)$ of the controls, marked inflammatory infiltration was present in $29 \%(11 / 37)$ of the malnourished group compared to none in the control group. A statistically significant difference $(p<0.05)$ was observed between malnourished and control groups in terms of villous height, crypt depth and inflammatory infiltration (Fig. 4).

\section{Discussion}


Our study revealed a high prevalence of chronic non-specific duodenitis diagnosed by histopathology in adults, irrespective of nutritional status. The finding is novel as there is no nationally representative data on duodenitis for this age group. A thorough literature search yielded very limited data on the prevalence of duodenitis from this geographic region. Though several studies exist that have investigated pathologies of the stomach and esophagus ${ }^{23}$, very few reported duodenal pathologies ${ }^{23}$. The findings conform to a recent study which also showed high prevalence of duodenitis in two-year-old chronic malnourished slum-dwelling children in Dhaka ${ }^{24}$. A study in Sweden reported 32\% prevalence of duodenitis in adults with $\mathrm{GI}$ symptoms ${ }^{25}$, however, those participants were well-nourished and underwent UGI endoscopy for diagnostic purposes, whereas more than half of the population in the current study were malnourished and asymptomatic.

The most common etiology for duodenitis in Western countries is celiac disease (CD), accounting for as high as $32 \%{ }^{26}$. In our study, only one malnourished adult, who also fell in the category of specific duodenitis, was found positive for $C D$, and the IEL count, which is a key histopathologic marker of $C D^{27}$, was also insignificant in the studied population. Biopsy samples could not be collected from absolutely healthy, asymptomatic individuals, due to ethical considerations. The control group considered in the present study consisted of age and sex-matched well-nourished (BMI > 18.5) adults suffering from functional dyspepsia diagnosed by Rome III criteria who had no evidence of any organic diseases on endoscopy, clinical and biochemical assessment and were otherwise healthy ${ }^{15}$. A study conducted on Dutch population showed that $83 \%$ patients with functional dyspepsia with no evident organic disease had chronic non-specific duodenitis on histopathology ${ }^{28}$, which is similar to our findings in this study.

Comparing the histological characteristics of both malnourished and control groups showed significant difference in villous height, crypt depth and inflammatory infiltration. The malnourished adult group had significantly higher frequency of subtotal villous atrophy, crypt hyperplasia and marked cellular infiltration $(p<0.05)$ than the controls. On the other hand, the control group had significantly higher prevalence of normal villous height, normal crypt depth and mild form of inflammation compared to the malnourished group $(p<0.05)$. Most importantly, the frequency of chronic active duodenitis was significantly high in malnourished population $(p<0.05)$ compared to the control group. As already mentioned these malnourished adults failed to respond to nutritional therapy and were also negative for any other secondary causes of malnutrition and thus were considered as probable cases of EED. The active feature is found in the setting of a relatively long term chronic inflammatory condition, which in turn is characterized by features like atrophied villi, hyperplastic crypts, inflammatory cellular infiltrates etc. This might in part explain the reason of the high frequency of active duodenitis in the malnourished cohort. Then again, studies relating EED biomarkers with the histopathology results are required to establish this as a fact.

\section{Limitations}


This study was conducted as a part of the Bangladesh Environmental Enteric Dysfunction (BEED) study and the target population of the present study were malnourished $(\mathrm{BMI}<18.5 \mathrm{~kg} / \mathrm{m} 2)$ and well-nourished $(\mathrm{BMI}>18.5)$ adults aged $18-45$ years. As collection of small intestinal biopsy samples from absolutely healthy, well-nourished, asymptomatic individuals without an evident clinical condition is ethically infeasible, the control group considered in the present study consisted of age and sex-matched wellnourished apparently healthy adults suffering from functional dyspepsia with no evidence of any organic diseases on endoscopy and clinical assessment. Association of EED markers with gut histology was also not explored. This remained a limitation of the study.

\section{Conclusion}

The prevalence of chronic non-specific duodenitis in Bangladeshi adults, irrespective of nutritional status, was high. Malnourished adults had significantly higher frequency of subtotal villous atrophy, crypt hyperplasia and marked cellular infiltration than the controls, whereas, healthy adults had significantly higher prevalence of normal villous height, normal crypt depth and mild form of inflammation compared to the malnourished group. The prevalence of chronic active duodenitis was significantly high in the malnourished adults.

\section{Declarations}

\section{Data Availability}

The dataset generated and analyzed during the current study is available from the corresponding author on reasonable request.

\section{Acknowledgements}

icddr,b acknowledges with gratitude the commitment of Bill and Melinda Gates Foundation (BMGF) to its research efforts. We express our sincere thanks to our colleagues at icddr,b and the study participants. icddr,b acknowledges with gratitude the commitment of the University of Virginia, Washington University in St. Louis, Dhaka Medical College and Hospital, Bangabandhu Sheikh Mujib Medical University, Dhaka Shishu Hospital, Bangladesh Specialised Hospital and Apollo Hospital, Dhaka to its research efforts. icddr,b is also grateful to the Governments of Bangladesh, Canada, Sweden and the UK for providing core/unrestricted support.

\section{Authors' contributions}

TA originated the idea and led the protocol and MSH participated in the design of the study. MSH, MMR, RNM, MAG, MMH and SMF were involved in sample and data collection. MSH, SMKNB, and MP performed and supervised the sample analysis. MSH, SD and MM were involved in data analysis. MSH, SMKNB and TA interpreted the results. MSH, SMKNB, SAS and TA were involved in manuscript writing. All the authors read, reviewed and approved the final manuscript. 


\section{Trial registration number}

ClinicalTrials.gov ID: NCT02812615 (https://clinicaltrials.gov/ct2/results?

cond $=$ NCT02812615\&term $=\&$ cntry $=\&$ state $=\&$ city $=\&$ dist $=$ )

\section{Date of first registration: $24 / 06 / 2016$}

\section{Funding}

This work was supported by the Bill and Melinda Gates Foundation (BMGF) under its Global Health Program. The project investment ID is OPP1136751.

(https://www.gatesfoundation.org/How-We-Work/Quick-Links/Grants-

Database/Grants/2015/11/OPP1136751).

\section{Competing Interests}

The authors declare no competing interests.

\section{References}

1. Denno, D. M., VanBuskirk, K. M., Nelson, Z. C., Musser, C. A. \& Tarr, P. I. Chapter One. Environmental enteric dysfunction (EED) background. (2016).

2. Amadi, B. et al. Impaired barrier function and autoantibody generation in malnutrition enteropathy in Zambia. EBioMedicine 22, 191-199 (2017).

3. Keusch, G. T. et al. Implications of acquired environmental enteric dysfunction for growth and stunting in infants and children living in low-and middle-income countries. Food and nutrition bulletin 34, 357-364 (2013).

4. Lindenbaum, J., Gerson, C. D. \& Kent, T. H. Recovery of small-intestinal structure and function after residence in the tropics: I. Studies in Peace Corps Volunteers. Annals of Internal Medicine 74, 218$222(1971)$.

5. Gerson, C. D., Kent, T. H., Saha, J. R., Siddiqi, N. \& Lindenbaum, J. Recovery of Small-Intestinal Structure and Function After Residence in the Tropics: Il. Studies in Indians and Pakistanis Living in New York City. Annals of internal medicine 75, 41-48 (1971).

6. Louis-Auguste, J. et al. High dose multiple micronutrient supplementation improves villous morphology in environmental enteropathy without HIV enteropathy: results from a double-blind randomised placebo controlled trial in Zambian adults. BMC gastroenterology 14, 15 (2014).

7. Farràs, M. et al. Characterizing the metabolic phenotype of intestinal villus blunting in Zambian children with severe acute malnutrition and persistent diarrhea. PloS one 13, e0192092 (2018).

8. Harper, K. M., Mutasa, M., Prendergast, A. J., Humphrey, J. \& Manges, A. R. Environmental enteric dysfunction pathways and child stunting: A systematic review. PLoS neglected tropical diseases 12, 
e0006205 (2018).

9. Kelly, P. et al. Responses of small intestinal architecture and function over time to environmental factors in a tropical population. The American journal of tropical medicine and hygiene $70,412-419$ (2004).

10. Rogawski, E. T. \& Guerrant, R. L. The burden of enteropathy and "subclinical" infections. Pediatric Clinics 64, 815-836 (2017).

11. Crane, R. J., Jones, K. D. \& Berkley, J. A. Environmental enteric dysfunction: an overview. Food and nutrition bulletin 36, S76-S87 (2015).

12. Islam, A. \& Biswas, T. Chronic stunting among under-5 children in Bangladesh: A situation analysis. Adv Pediatr Res 2, 18 (2015).

13. Pryer, J. A. \& Rogers, S. Epidemiology of undernutrition in adults in Dhaka slum households, Bangladesh. European journal of clinical nutrition 60, 815 (2006).

14. Nube, M. \& Van Den Boom, G. Gender and adult undernutrition in developing countries. Annals of Human Biology 30, 520-537 (2003).

15. Mahfuz, M. et al. Bangladesh Environmental Enteric Dysfunction (BEED) study: protocol for a community-based intervention study to validate non-invasive biomarkers of environmental enteric dysfunction. BMJ open 7, e017768 (2017).

16. Van Der Hulst, R. R. et al. Gut permeability, intestinal morphology, and nutritional depletion. Nutrition 14, 1-6 (1998).

17. Whitehead, R., Roca, M., Meikle, D., Skinner, J. \& Truelove, S. The histological classification of duodenitis in fibreoptic biopsy specimens. Digestion 13, 129-136 (1975).

18. Wang, C.-X., Liu, L.-J., Guan, J. \& Zhao, X.-L. Ultrastructural changes in non-specific duodenitis. World Journal of Gastroenterology: WJG 11, 686 (2005).

19. Caselli, M. et al. Histologic findings and Helicobacter pylori in duodenal biopsies. Journal of clinical gastroenterology 26, 74-80 (1998).

20. Kelly, P. et al. Enteropathy in Zambians with HIV related diarrhoea: regression modelling of potential determinants of mucosal damage. Gut 41, 811-816 (1997).

21. Kelly, P. et al. Endomicroscopic and transcriptomic analysis of impaired barrier function and malabsorption in environmental enteropathy. PLoS neglected tropical diseases 10, e0004600 (2016).

22. Serra, S. \& Jani, P. A. An approach to duodenal biopsies. Journal of clinical pathology 59, 1133-1150 (2006).

23. Akbulut, U. E., Fidan, S., Emeksiz, H. C. \& Ors, O. P. Duodenal pathologies in children: a single-center experience. J Pediatr (Rio J), doi:10.1016/j.jped.2017.06.018 (2017).

24. Hossain, M. S. et al. Asymptomatic Duodenitis and Helicobacter pylori associated Dyspepsia in 2Year-Old Chronic Malnourished Bangladeshi Slum-Dwelling Children: A Cross-Sectional Study. Journal of Tropical Pediatrics (2020). 
25. Borch, K. et al. Prevalence of gastroduodenitis and Helicobacter pylori infection in a general population sample. Digestive diseases and sciences 45, 1322-1329 (2000).

26. Alper, A. et al. Prevalence and Clinical, Endoscopic, and Pathological Features of Duodenitis in Children. J Pediatr Gastroenterol Nutr 62, 314-316, doi:10.1097/MPG.0000000000000942 (2016).

27. Oberhuber, G., Granditsch, G. \& Vogelsang, H. The histopathology of coeliac disease: time for a standardized report scheme for pathologists. European journal of gastroenterology \& hepatology 11 , 1185-1194 (1999).

28. Kreuning, J., Wal, A., Kuiper, G. \& Lindeman, J. Chronic nonspecific duodenitis. A multiple biopsy study of the duodenal bulb in health and disease. Scandinavian Journal of Gastroenterology 24, $16-$ 20 (1989).

\section{Tables}

Table 1

Distribution of duodenitis based on the operational classification

\begin{tabular}{|llll|}
\hline Duodenitis & Adult Cohort & \\
\hline & $\begin{array}{l}\text { Malnourished } \\
+n=37 \\
\text { tn (\%) }\end{array}$ & $\begin{array}{c}\text { Control } \\
+n=27 \\
+n(\%)\end{array}$ & †P-value \\
& & & \\
\hline Based on known pathology & $0(0 \%)$ & $1(6 \%)$ & 0.42 \\
\hline Normal & $2(5 \%)$ & $0(0 \%)$ & 0.50 \\
\hline Specific duodenitis & $35(95 \%)$ & $26(96 \%)$ & 1.00 \\
\hline Non-specific duodenitis & & & \\
\hline Based on tissue morphology & $0(0 \%)$ & $1(6 \%)$ & 0.42 \\
\hline Normal & $12(32 \%)$ & $18(66 \%)$ & 0.007 \\
\hline Chronic duodenitis & $25(68 \%)$ & $8(30 \%)$ & 0.003 \\
\hline Chronic active duodenitis & $2(5 \%)$ & $0(0 \%)$ & 0.50 \\
\hline Intraepithelial lymphocytosis (†IEL > 30) & & & \\
\hline † n, Number of respondents; P-value, Significance level; IEL, Intraepithelial lymphocytosis & \\
\hline
\end{tabular}

\section{Figures}




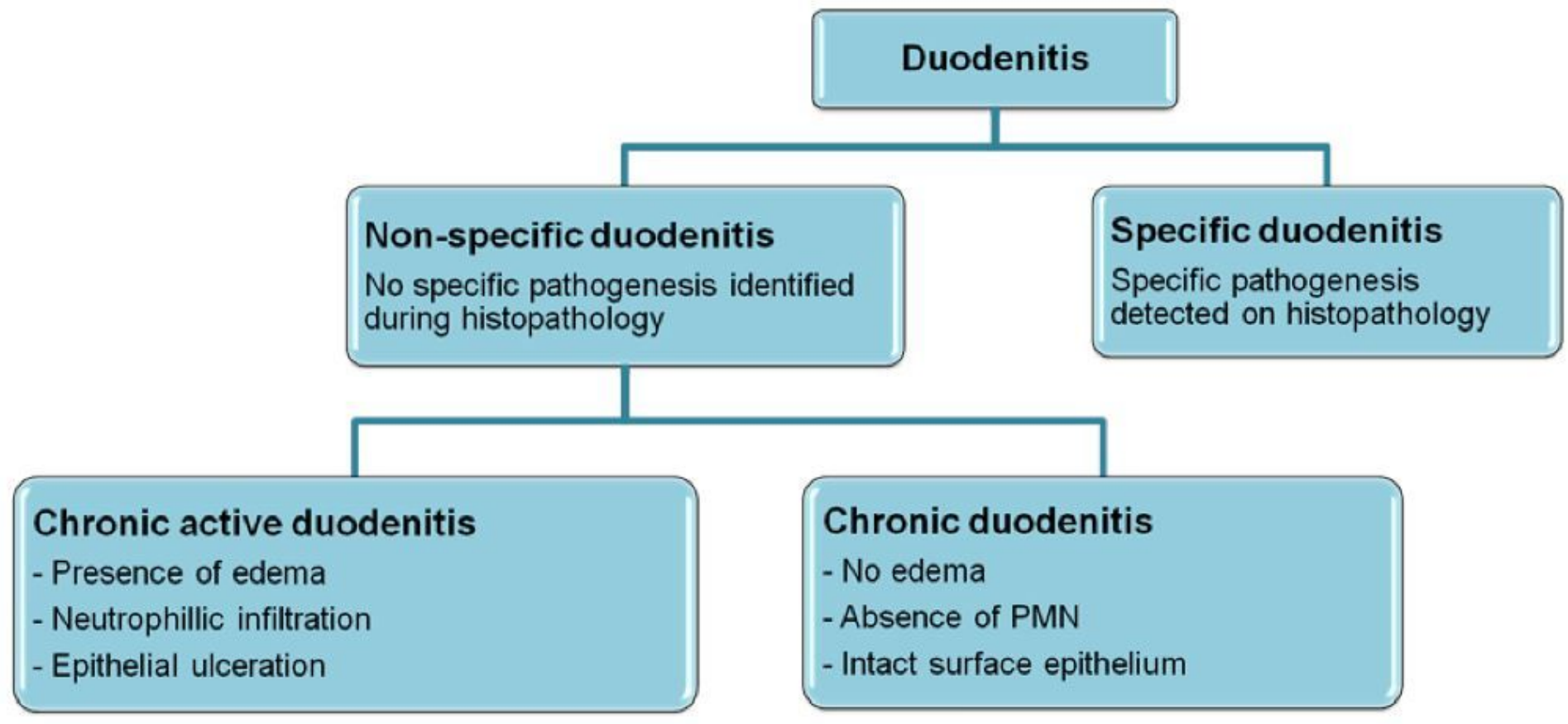

Figure 1

Caption: Operational classification of duodenitis based on pathogenesis and cellular activity Legends: Duodenitis; Non-specific duodenitis; No specific pathogenesis identified during histopathology; Specific duodenitis; Specific pathogenesis identified on histopathology; Chronic active duodenitis; Presence of edema; Neutrophillic infiltration; Epithelial ulceration; Chronic duodenitis; No edema; Absence of *PMN; Intact surface epithelium. *PMN, Polymorphonuclear leukocytes.
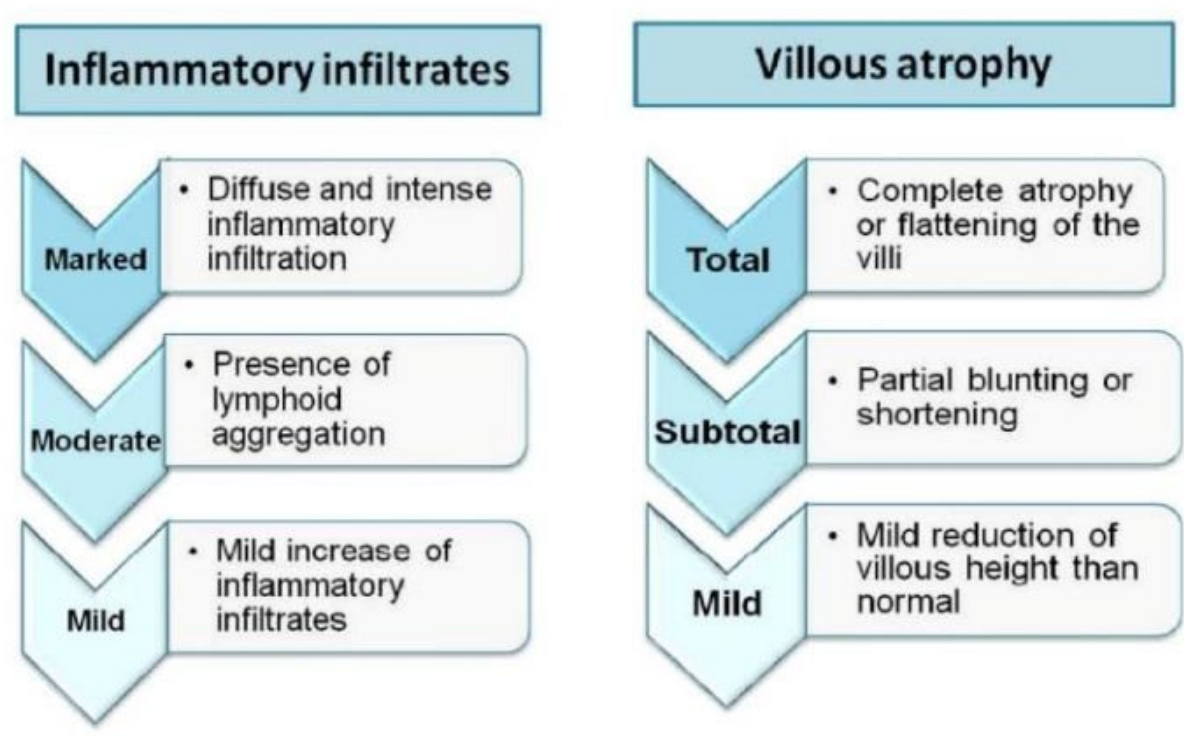

\section{Crypt hyperplasia}

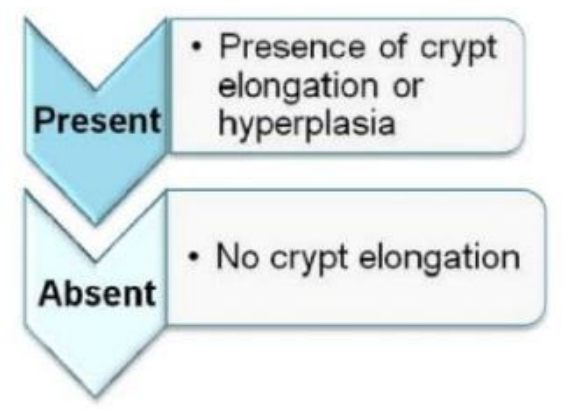

Figure 2 
Caption: Schematic presentation for assessment of the histological alterations of intestinal mucosa Legends: Inflammatory infiltrates; Marked; Diffuse and intense inflammatory infiltration; Moderate; Presence of lymphoid aggregation; Mild; Mild increase of inflammatory infiltrates; Villous atrophy; Total; Complete atrophy or flattening of the villi; Subtotal; Partial blunting or shortening; Mild; Mild reduction of villous height than normal; Crypt hyperplasia; Present; Presence of crypt elongation or hyperplasia; Absent; No crypt elongation.
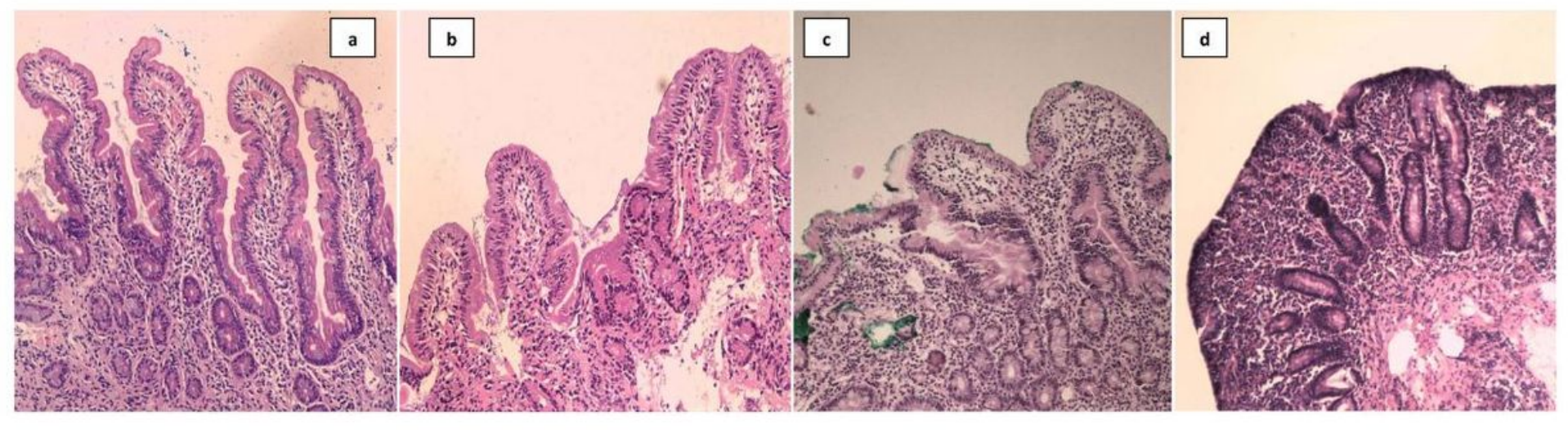

\section{Figure 3}

Caption: Histological images of villous height obtained using hematoxylin and eosin (H\&E) stain Legends: a. Normal villous architecture b. Mild villous atrophy c. Subtotal villous atrophy d. Total villous atrophy with crypt hyperplasia 


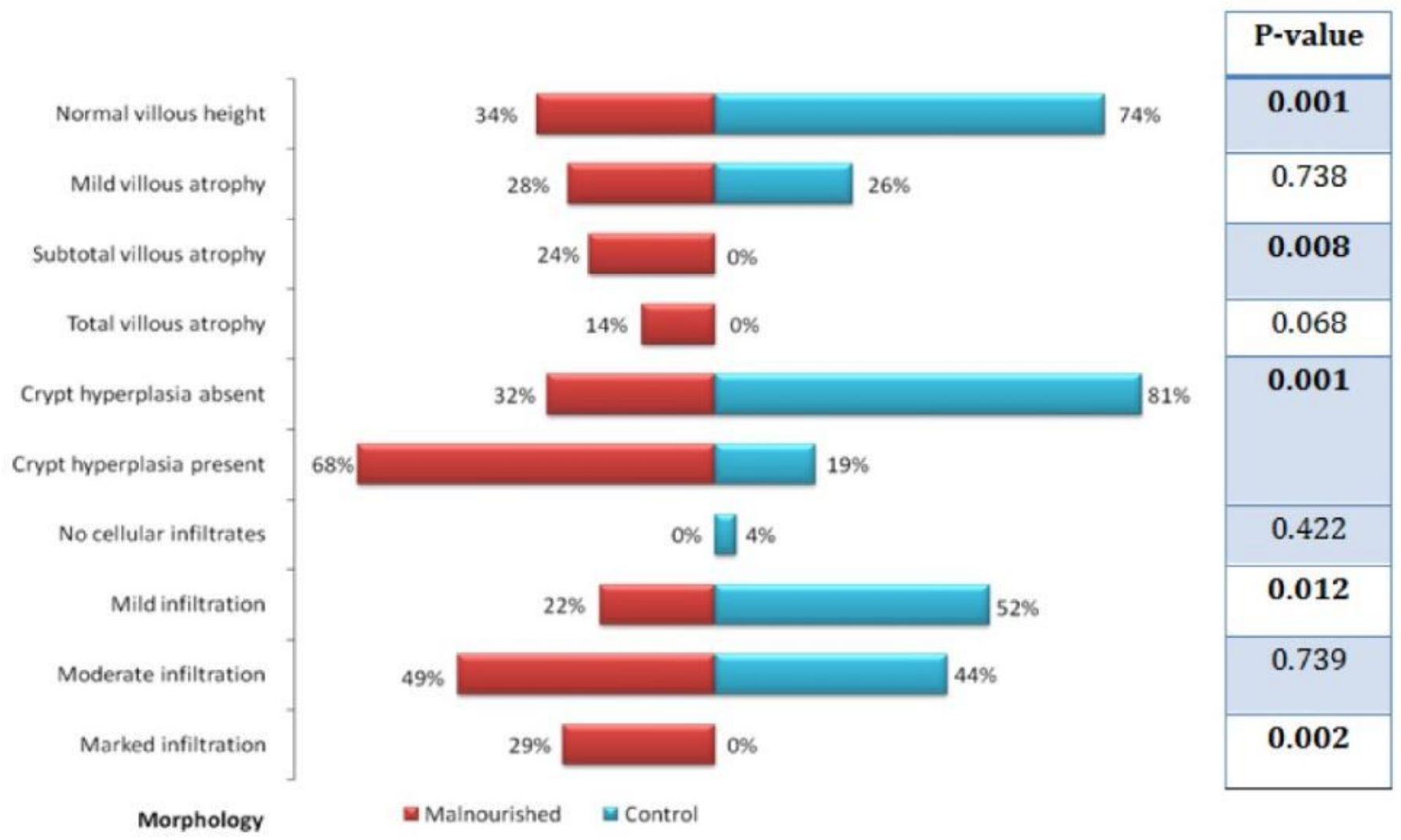

Figure 4

Caption: Comparison of histological features between malnourished and healthy adults Legends: Normal villous height; Mild villous atrophy; Subtotal villous atrophy; Total villous atrophy; Crypt hyperplasia absent; Crypt hyperplasia present; No cellular infiltrates; Mild infiltration; Moderate infiltration; Marked infiltration; Morphology; Malnourished; Control; *P-value. *P-value, Significance level 